\title{
Observations of the IMF in clusters
}

\author{
Joana Ascenso ${ }^{1}$ \\ ${ }^{1}$ Centro de Astrofísica da Universidade do Porto, Rua das Estrelas, 4150-762 Porto, Portugal \\ email: jascenso@astro.up.pt
}

\begin{abstract}
Stars form from molecular clouds, mostly in clusters with tens to tens of thousands of members, and the mass distribution within these clusters, or the Initial Mass Function, seems to be invariable against many parameters and over a wide range of masses. However, masses are a very difficult quantity to assess, and the precision of our determinations of the IMF is systematically lower than usually quoted. I will discuss the process of determining masses from observations and the type of uncertainties associated with this process.
\end{abstract}

Keywords. techniques: photometric, stars: fundamental parameters, stars: luminosity function, mass function, (Galaxy:) open clusters and associations: general

\section{Introduction}

The Initial Mass Function (IMF) describes the distribution of stellar masses at birth for a single star formation event. The significance of understanding the IMF is transversal to most fields of modern astronomy: not only does it help to constrain the process of star formation, but it is also all important as an input parameter for studies of galactic populations and evolution, chemistry of the interstellar (and intergalactic) medium, and cosmology (e.g., the STARBURST99 code, by Leitherer et al. (1999), has been used extensively in studies based on assumptions of the IMF).

The IMF was first introduced by Salpeter in 1955 when characterizing the field population of the Galaxy, and it was then defined as a power-law of index -1.35 , since known as the Salpeter slope. In the decades that followed, the IMF was measured in all possible environments, including stellar clusters and associations featuring a wide range of ages, age ranges, masses, stellar densities, morphologies, massive star content and metallicities, both in our Galaxy and in other galaxies, some of which with intense starburst activity (e.g. Bastian et al. 2010, and references therein). These studies found that the Salpeter power-law breaks around a characteristic mass of $0.3-0.8 \mathrm{M}_{\odot}$, and the IMF becomes flatter, although still rising, until it starts to decline in the substellar regime (Kroupa 2001; Chabrier 2003). Although the exact form of the subsolar IMF is still a matter of active debate, all observational evidence suggests the IMF is invariable over a wide range of densities, environments, and metallicities, at least in the high-mass range. This is becoming more and more clear as new studies using more sophisticated technology and methods reveal "universal" IMFs even in regions previously said to have peculiar mass functions (e.g. Espinoza et al. 2009).

Given the predominance of non-observers in this meeting, I chose to focus on the actual process of determining the IMF from observations rather than reviewing the actual results. The reader is referred to the recent excellent reviews of the "observed" IMF by, e.g., Bastian et al. (2010); Elmegreen (2009); Clarke (2009); Elmegreen (2005); Chabrier (2005); Luhman (2004); Lada \& Lada (2003).

This contribution is not meant to describe all possible ways to solve the main steps toward determining masses from observations. Instead, I will outline the process in moderate detail describing the most commonly used techniques, while emphasizing the 
associated difficulties and challenges, and conclude with the argument that, in face of so many, often unquantifiable, sources of uncertainty, it is only expected that the IMF shows scatter from region to region, even if it is universal.

\subsection{The IMF in clusters}

Stellar clusters are widely accepted as units of star formation. They are the immediate outcome of the process of star formation from molecular clouds, and as such are the ideal end-product probes of the initial conditions for star formation in the current era of nearinfrared telescopes and surveys (Lada \& Lada 2003). The main advantages of studying the IMF in young clusters follow from their group nature: they are statistically significant samples of coeval, equidistant, and volume-limited stars that share the properties of a common parent molecular cloud, such as metallicity, and initial conditions (pressure, temperature, ambient radiation, magnetic fields, etc.), and are thus ideally suited for the study of a group property such as the IMF. Although the IMF was studied thoroughly in all types of clusters - globular clusters (a few Gyr), open clusters (a few $\times 10$ Myr), and embedded clusters (a few Myr) -, the latter, embedded clusters, present the most favorable conditions for the characterization of the IMF. Being the youngest, their present-day mass function is closest to their initial mass function, as they have not yet had time to lose members to stellar or dynamical evolution, and so no additional corrections, often sources of uncertainty, are needed. Also, their young stars are still bright in the near-infrared, and the stellar mass spectrum presents a much smaller dynamic range in brightness when compared to equivalent observations in the optical, which favors the completeness of the observed samples. Finally, their compactness and embedded state provide a natural shield against background contamination by unrelated sources, thus minimizing a significant potential bias when studying the properties of the cluster.

For these reasons, I will focus on observations of the IMF in young clusters, rather than reviewing the measurements done in all other environments. Many of the methods and challenges are, nonetheless, common to all determinations of mass.

\subsection{Measuring the IMF}

The determination of the IMF of any given population depends on our ability to determine masses. Unfortunately, mass is not a direct observable, hence the difficulty of deriving accurate and consistent IMFs for different regions. The determination of stellar masses relies heavily on assumptions, on models, and on the knowledge of other properties like distance and age that are, by themselves, very challenging to ascertain.

The most accurate way to derive stellar masses is through high-resolution spectroscopy, preferably associated to photometric data, covering a wide range of wavelengths. Spectra allow for the determination of spectral type and evolutionary status of individual stars. Single-band photometry, when combined with the spectral information, helps to assess membership and to determine the distance, whereas the multi-wavelength coverage allows for the construction of a spectral energy distribution, leading to the characterization of the circumstellar material around the star. The spectroscopic approach, although reliable, assuming our knowledge of pre-main-sequence spectral features is accurate, is extremely inefficient and expensive in telescope time. It requires each cluster member to be observed individually, in many bands, and using different instruments and setups, preferentially simultaneously to prevent errors associated with the ubiquitous variability of young stars. Also, spectroscopy of the faint members requires long integration times, even in the largest telescopes, all translating into impractical prerequisites for clusters with more than a few members and more distant than just a few kpc. 
The best alternative is to use broad-band photometry in several wavelengths and infer the properties of the individual stars based on their brightness and color. This approach is relatively inexpensive, as it provides useful information about all cluster members in just a few images with the same telescope time investment, meaning less telescope time for more stars. Photometry, by definition, will also reach fainter stars with shorter integration times when compared to spectroscopy. The drawback is that the information given by broad-band photometry is limited, and more needs to be inferred. Using the brightness and color of each cluster member, one can construct a luminosity function that can then be transformed into a mass function using the appropriate mass-luminosity relation. Often, it pays to have spectra of at least the few brightest members to help constrain the distance and age of the cluster, assuming coevality.

In the following sections I will describe the difficulties associated with deriving the IMF from broad-band, multi-wavelength photometry of young, embedded clusters.

\section{Observational challenges}

\subsection{Membership assessment}

Most molecular clouds and young clusters of our Galaxy are located in the galactic disc. Stars from the field, that are physically unrelated with the cluster, are therefore likely to be present in any image of a cluster, and to be included in the sample as part of the cluster population. But including these stars in the sample is equivalent to observing a mass function that is the convolution of the field present-day mass function with the cluster IMF, worsened by the fact that we will most likely detect a disproportionate amount of background giants, for example, if one is observing in the infrared. Discerning which stars in an image are actually physically associated with the cluster is therefore determinant to an accurate analysis of the cluster properties, and in particular of the IMF.

There are several ways to disentangle the cluster stars from the foreground/background contaminants, each requiring specific observations and analysis. One of the most accurate methods of assessing membership relies on proper motion studies: by observing the cluster at several epochs so that its stars can be seen moving against the background, one can measure the projected velocity of the individual stars. Assuming that the cluster members have similar velocities it is possible to establish the membership of each star individually. Although accurate, this method often requires prohibitively large time baselines, especially if the region of interest is distant, and multiple very high-resolution observations of the same region, which is not easy to obtain given the constraints in telescope time.

The previous method is most frequently used for nearby open clusters (e.g., Caballero 2010; Krone-Martins et al. 2010), where membership assessment is hardest. In young, embedded clusters, although it would still be possible to use the dynamical method described above (e.g. Stolte et al. 2008), there are other, less expensive options that can simultaneously be applied to clusters farther away from the Sun. If a cluster is embedded, then the foreground objects will be at "zero" extinction, making them relatively easy to identify. Similarly, the few background stars that will still be detected through the cloud will be behind an extinction "wall", allowing for their identification, in principle, based on extinction arguments. However, all cluster members do not present one single value of extinction, but rather a continuum of extinction values around a mean deriving from the patchy nature of the cloud, making it difficult to determine at which value of extinction a star is no longer a member of the cluster. Also, if the cloud is only thick enough to 
embed the cluster, then the shielding of background sources becomes less effective. Using ages - most cluster stars are still on the pre-main-sequence, making their identification less challenging using multi-band photometry alone and/or X-ray data - and the related presence of circumstellar disks, one can furthermore separate out the young from the old, main-sequence field objects in the image, and attribute membership to all objects that match a reasonable age for the cluster. Moreover, not all stars will have circumstellar material, nor will they be all in the pre-main-sequence, making the age assessment more difficult.

For these reasons, most studies use statistical methods instead to subtract the contamination from field stars, by observing a nearby control field at the same galactic latitude with the exact same setup. Once properly reddened by the same amount of (mean) extinction or, if available, by the extinction profile of the cloud, this sample becomes a good statistical representation of the foreground/background population toward the science field. Since the IMF is most commonly derived from a single-band luminosity function, one can statistically subtract a reddened control-field population from the science sample, thus correcting for contamination. Although it does not provide individual memberships, this method does provide a reasonable correction from contaminants for IMF purposes.

\subsection{Completeness}

Our ability to detect all stars in a cluster determines the accuracy of our IMF. Stars can go undetected for several reasons, both observational and characteristic of the star forming region itself. In this section I will describe the various parameters that can affect the completeness of the observations, and ultimately bias the IMF.

\subsubsection{Sensitivity}

The most obvious consequence of having time-limited observations is that we cannot detect objects fainter than some brightness limit. Due to exposure time and to the intrinsic characteristics of the detector, we will only be sensitive to signal that falls above some brightness threshold, compromising our ability to sample the IMF to the lowest mass range. To a first approximation, knowing the completeness limit of a sample will delimit the mass range over which one feels confident that the IMF is fully sampled, but many authors use this information further to correct their sample of incompleteness by dividing the observed mass function by the completeness profile (e.g. Stolte et al. 2002, but see also discussion in Ascenso et al. (2009)).

The best way to estimate the completeness of a given sample is to perform artificial stars experiments. These consist in adding artificial stars of different brightnesses to the observed cluster image and trying to recover them with the correct brightness using the exact same method used for the detection and photometry of the science stars (Ascenso et al. 2009). This is done in most studies of clusters and the IMF, the exact details of the method varying from study to study.

\subsubsection{Resolution}

Despite the great advances in the past decades, resolution is still a limiting factor in modern observations. The farthest and the denser the star forming region, the more difficult it is to have a comprehensive view of the full stellar population, even above the sensitivity limit. The first consequence of not being able to resolve (visual) multiples is that the luminosities of "individual" objects, that actually comprise two or more stars, are overestimated, counting as more massive stars. Although it is not a big increase in brightness, and therefore in mass, for a, say, $20 \mathrm{M}_{\odot}$ star to be blended with a low-mass 
object, it will make a significant difference for a low-mass object to be blended with an equal mass object, especially if one considers that the mass bins of the IMF are usually logarithmic. The other consequence is one of demographics: blending stars means we lose cluster members. This incompleteness effect is mass-dependent, and thus may bias the IMF.

The most advanced observatories and space telescopes are less affected by this problem, the first due to adaptive optics technology, and the latter to being above the atmosphere, being almost only limited by diffraction. As before, the best solution to blending would be to analyse all stars spectroscopically, looking for signatures of unresolved neighbors. Alternatively, by performing artificial star experiments, one can correct the sample globally (c.f. 2.2.1).

\subsubsection{Variable extinction}

Perhaps the major downside of having a cluster embedded in a molecular cloud is, since the cloud is often patchy, that each star is likely subject to a different amount of extinction. This causes problems of variable completeness in the field, as the extinction dims the brightness of the stars, inevitably causing some to fall below the detection limit. This effect is more important for the youngest clusters, where the cloud is still well within the cluster, permeating the intracluster medium. As the cluster evolves and the stars clear most of the gaseous and dusty material this problem becomes less significant.

The effect of variable extinction in a cluster is somehow a function of mass: whereas the brightest, more massive stars will still be detected as reddened objects, the faint, low-mass stars will most likely be dimmed away from detection, causing the IMF to be incomplete toward the lower-mass end. This effect is very difficult to quantify without an extinction map of the region, which is usually itself very challenging to obtain for regions of star formation since there is not a clear view of the background stars. Since it is possible to estimate the extinction to each individual detected object, one can at most use that information to estimate which mass bins would most likely be affected by the variable extinction. This would always be an incomplete assessment, as one can only measure the extinction caused by the material in front of any specific star, and not the full length of extinction any cluster star is potentially subject to.

\subsubsection{Variable nebula}

Similar to the effect of variable extinction is the effect of having a bright nebula of varying intensity. This is seen frequently in embedded clusters with one or several bright, massive stars that ionise the gas in the intracluster medium, making it bright. The nebula then acts as an extended emission that lowers our ability to detect faint stars by reducing the contrast between the background and the object.

Again, this affects mostly the low-mass end of the mass spectrum, but, unlike the incompleteness due to variable extinction, this can be quantified, namely using the artificialstar completeness tests mentioned above. However, given the usually patchy nature of the nebula, and the fact that the brightness may vary significantly from region to region, the statistical approach of the artificial stars experiments may not be effective as a correction tool: if the areas affected by the nebula are locally small, then it is likely that they are hiding only a small number of stars, making the correction too susceptible to small number statistics.

\subsection{Interstellar Extinction and Circumstellar Material}

Estimating the mass of any embedded source requires, among other things, that the extinction is known or can be derived. Using multi-band photometric observations, this 
can be done by comparing the color of an object with that of a similar, unreddened object. This implies that we know two things: (1) the underlying, intrinsic nature of the object, and (2) the way extinction acts on brightness and color, i.e., the extinction law.

Determining the intrinsic color of a young object is always a challenge, both because of extinction, and because, depending on the exact age of the cluster, low-mass objects will have circumstellar discs and/or envelopes that increase the object's brightness and further redden its colors. The reddening from the circumstellar material, however, acts on a different direction in a color-color diagram than does the interstellar reddening, so it is possible, at least to some degree, to disentangle the two. Determining the amount of excess emission from an object is crucial to estimating its mass: if a star appears brighter than it actually is due to emission from circumstellar material, its mass will be overestimated, potentially biasing the IMF. To this end, spectroscopy and/or the analysis of the spectral energy distribution (SED) of each object helps to determine the fraction of light that is emitted by the circumstellar material, although building an SED also requires having observations covering a large spectral range, while spectroscopy of many objects is prohibitively time-consuming. The solution is often to de-excess and de-redden each object by, first, roughly estimating the emission from circumstellar material using empirical models (Meyer et al. 1997), and then assume (pre-)main-sequence photosphere colors as comparison standards to estimate the extinction.

This last step requires the knowledge of the extinction law. For a long time believed to be universal, it has recently been found that the law may be density-dependent (e.g, Román-Zúniga et al. 2007). The relevant departures from the "universal" law have mostly been found in the mid-infrared regime, and have been proposed to originate in different grain composition and size: in denser areas, the grains are expected to grow and coalesce thus producing different signatures in the extinction properties. The near-infrared extinction law appears not to be significantly affected by density. Ideally, one would derive the extinction law for each specific region of star formation, but this would require access to a pristine background population in the direction of the cluster rather than the typical zoo of young objects, ionizing nebula, and reddened objects typically found toward a star forming region. The "universal" law (Rieke \& Lebofsky 1985) is still used in most studies of embedded clusters.

\subsection{Mass-luminosity relation}

The last step toward a catalog of masses is to derive the actual masses from the observed luminosities. The difficulty here is to find suitable models and have a good estimate of the age of the stars. Massive and low-mass stars evolve on different timescales, so, even in the (unlikely) case of a perfectly coeval population, there will still be massive main-sequence stars, and lower-mass pre-main-sequence objects, the exact fraction of which depending on the age. We must therefore use a combination of both main-sequence (e.g., Lejeune \& Schaerer 2001) and pre-main-sequence models (e.g., Palla \& Stahler 1993; Baraffe et al. 1998), adjusted to whatever age and distance we derive for the cluster. Although the current models for pre-main-sequence objects still have many uncertainties, age and distance estimates are by far the greatest sources of error in the determination of the mass-luminosity relation, and therefore, in the determination of mass.

\subsubsection{Age}

Age estimates rely on placing the cluster members on HR diagrams, and comparing their position with theoretical evolutionary tracks. This is usually done using color information and/or spectra of individual stars, with the caveat that, as mentioned before, the presence of circumstellar material can produce deceiving colors and lead to 
erroneous age estimates, not to mention the effect on the mass determination itself. The pre-main-sequence models are continuously being improved, and already seem to capture the essence of pre-main-sequence objects, but factors like accretion have been shown to have a high impact on the models (Hartmann et al. 1997; Baraffe et al. 2009).

The presence of short-lived, massive pre-main-sequence stars in the cluster can also constrain the age if one assumes coevality. The expected lifetime of a massive star in the main-sequence can, in this case, be used as an upper limit for the cluster age. Other youth indicators may also help constrain the approximate age of the cluster. It is expected that the embedded phase lasts 3 to 5 Myr (Lada \& Lada 2003), during which time the stars will effectively clear the remains of the molecular cloud. The existence of class I sources, the presence of jets, or phenomena, such as proplyds, believed to be short-lived, also testify to youth. A linear relation of disc frequency with age has been proposed by Haisch et al. (2001), but it is not yet solid enough to serve as a reliable constraint (Mayne et al. 2007). In any case, one can never expect to be accurate to more than 1 or $2 \mathrm{Myr}$, at best.

Once the age is known to whatever accuracy, it is possible to estimate which fraction of stars are likely main-sequence, and which are pre-main-sequence, but even then, there is still some degeneracy, in that two pre-main-sequence stars may have the same brightness even though their mass is (slightly) different (see discussion in Ascenso et al. 2007). This degeneracy could, in principle, be lifted by the colors, but the differences will most often be too subtle to prevail over observational errors, extinction, and circumstellar material. Finally, the mass-luminosity relation for subsolar-mass objects is still very poorly characterized, which would generate further uncertainty in this mass range even if the age was accurately known.

\subsubsection{Distance}

Distance, on its turn, can be constrained using either trigonometric parallax, for nearby regions, or, most frequently, spectroscopic parallax. The latter consists in comparing the observed brightness with that expected for a star, with its spectroscopically-derived spectral type. Alternatively, the distance can be constrained using dynamical arguments and models of the Galaxy to derive "kinematic distances" (Roman-Duval et al. 2009), or even using the density of foreground stars to a molecular cloud or cluster (Lombardi et al. 2010).

\subsection{What is a cluster?}

If one defines the IMF as the initial mass function of a single event of star formation, then one would need to observe all stars from one cluster or entire cloud in order to have a good representation of the IMF, depending on the definition of "single event of star formation" they choose. However, a cluster is neither believed to be coeval, nor does it have defined boundaries. This somewhat more philosophical question goes back to the understanding of the very process of star formation, either in clusters or in (relative) isolation throughout the cloud. Since clusters are (still?) believed to be the basic units of star formation, and given the constraints on telescope time, most studies of young populations have analysed single clusters rather than entire clouds, although the paradigm seems to be changing with the improvement of large surveys (e.g., Jørgensen et al. 2006). The question of cluster boundaries is pertinent even if one simplifies the analysis by considering single clusters: some numerical simulations of star forming clouds suggest that, along with the identifiable cluster, a significant population of stars formed in the same event are scattered over large areas (Bonnell et al. 2010). Rapid dynamical phenomena have also been theoretically described by which a cluster can rapidly disperse a fraction of its members (Binney \& 
Tremaine 1987). The gas removal, around 3 to 5 Myr into the cluster's age, will also unbound the cluster, leading to a rapid and effective scattering of cluster members over large areas around the identifiable cluster, or to the disruption of the cluster altogether (Bastian \& Goodwin 2006). All these possibilities are somehow mass-specific, introducing potential systematic errors when typically observing and characterizing the IMF of single clusters. This problem will be more significant the more effective these processes are in scattering the cluster population spatially, but will be less important the denser and more massive the cluster is, as most of its mass and members will, in principle, reside in the identifiable and gravitationally more bound cluster core.

Large multi-wavelength surveys are and will continue to be helpful in identifying cluster members residing outside the core, thus contributing immensely to our understanding of the star formation process.

\section{Technical challenges}

Finally, after having a trusted sample of masses, one needs to characterize the functional form of the mass distribution. The two most widely used IMFs are that of Chabrier (2003), consisting of a (Salpeter) power-law above $\sim 1 \mathrm{M}_{\odot}$ and a log-normal for lower masses; and that of Kroupa (2001), consisting of a three-segment power-law. Both are quasi-equivalent in shape and parameters.

These functions are usually fitted to a histogram of masses, obtained, traditionally, by binning the masses in equal-width dex bins, but this approach has been shown to be biased and should therefore be avoided (Maíz Apellániz \& Úbeda 2005). Instead, and according to these authors, the IMF must be built using bins with equal number of stars, therefore dissipating the bias entirely. Alternatively, one can use the modified maximum likelihood method proposed recently by Maschberger \& Kroupa (2009).

The fitting technique itself would be a matter of a whole different paper entirely and will not be addressed here.

\section{Summary}

This contribution describes the difficulties in deriving masses and mass functions from observational data, with special emphasis on the problematics of young clusters. Considering all these sources of uncertainty, it is probably not surprising that the initial mass function has turned out to be universal, even when measured in such different environments. On the other hand, even considering the uncertainties, it is clear that it also cannot be completely different from region to region. Although we are not (yet) in position to say that the IMF is strictly universal, observations continue to show a consistent overall shape of the IMF, as well as a consistent characteristic mass, hinting at some degree of universality reflecting the process of star formation.

It is clear from the literature that we are still struggling with methodology and instrument limitation issues, as one sometimes sees the same region of star formation showing different results with every new publication, but convergence seems to be closer than ever, especially for the high-mass end. Although benefitting from the lessons learned for the high-masses over the last six decades, the subsolar mass function is still taking its first serious steps (e.g., Luhman et al. 2009). Although naturally more prone to error and uncertainties, new, deep surveys of entire clouds are revealing the lower-mass end of star formation with unprecedented completeness and detail. The first results already hint at some consistency also in this regime, but the jury is still out on whether the low-mass end of the IMF is as universal as the high-mass end appears to be. 


\section{References}

Ascenso, J., Alves, J., Beletsky, Y., \& Lago, M. T. V. T. 2007, A\& A, 466, 137

Ascenso, J., Alves, J., \& Lago, M. T. V. T. 2009, A\&SA, 495, 147

Baraffe, I., Chabrier, G., Allard, F., \& Hauschildt, P. H. 1998, A\&A, 337, 403

Baraffe, I., Chabrier, G., \& Gallardo, J. 2009, ApJ, 702, L27

Bastian, N., Covey, K. R., \& Meyer, M. R. 2010, ARA\&A, 48, 339

Bastian, N. \& Goodwin, S. P. 2006, MNRAS, 369, L9

Binney, J. \& Tremaine, S. 1987, Galactic dynamics (Princeton, NJ, Princeton University Press, 1987, 747 p.)

Bonnell, I. A., Smith, R. J., Clark, P. C., \& Bate, M. R. 2010, ArXiv e-prints

Caballero, J. A. 2010, A\&A, 514, A18+

Chabrier, G. 2003, PASP, 115, 763

Chabrier, G. 2005, in Astrophysics and Space Science Library, Vol. 327, The Initial Mass Function 50 Years Later, ed. E. Corbelli, F. Palla, \& H. Zinnecker, 41-+

Clarke, C. J. 2009, ApESSS, 324, 121

Elmegreen, B. G. 2005, in Astrophysics and Space Science Library, Vol. 329, Starbursts: From 30 Doradus to Lyman Break Galaxies, ed. R. de Grijs \& R. M. González Delgado, 57-+

Elmegreen, B. G. 2009, in The Evolving ISM in the Milky Way and Nearby Galaxies

Espinoza, P., Selman, F. J., \& Melnick, J. 2009, A $\& A$, 501, 563

Haisch, Jr., K. E., Lada, E. A., \& Lada, C. J. 2001, ApJ, 553, L153

Hartmann, L., Cassen, P., \& Kenyon, S. J. 1997, ApJ, 475, 770

Jørgensen, J. K., Harvey, P. M., Evans, II, N. J., et al. 2006, ApJ, 645, 1246

Krone-Martins, A., Soubiran, C., Ducourant, C., Teixeira, R., \& Le Campion, J. F. 2010, A\&A, $516, \mathrm{~A} 3+$

Kroupa, P. 2001, MNRAS, 322, 231

Lada, C. J. \& Lada, E. A. 2003, ARA\&A, 41, 57

Leitherer, C., Schaerer, D., Goldader, J. D., et al. 1999, ApJs, 123, 3

Lejeune, T. \& Schaerer, D. 2001, A\&AA, 366, 538

Lombardi, M., Lada, C. J., \& Alves, J. 2010, A\&A, 512, A67+

Luhman, K. L. 2004, in IAU Symposium, Vol. 221, Star Formation at High Angular Resolution, ed. M. G. Burton, R. Jayawardhana, \& T. L. Bourke, 237-+

Luhman, K. L., Mamajek, E. E., Allen, P. R., \& Cruz, K. L. 2009, ApJ, 703, 399

Maíz Apellániz, J. \& Úbeda, L. 2005, ApJ, 629, 873

Maschberger, T. \& Kroupa, P. 2009, MNRAS, 395, 931

Mayne, N. J., Naylor, T., Littlefair, S. P., Saunders, E. S., \& Jeffries, R. D. 2007, MNRAS, 375, 1220

Meyer, M. R., Calvet, N., \& Hillenbrand, L. A. 1997, AJ, 114, 288

Palla, F. \& Stahler, S. W. 1993, ApJ, 418, 414

Rieke, G. H. \& Lebofsky, M. J. 1985, ApJ, 288, 618

Roman-Duval, J., Jackson, J. M., Heyer, M., et al. 2009, ApJ, 699, 1153

Román-Zúñiga, C. G., Lada, C. J., Muench, A., \& Alves, J. F. 2007, ApJ, 664, 357

Salpeter, E. E. 1955, ApJ, 121, 161

Stolte, A., Ghez, A. M., Morris, M., et al. 2008, ApJ, 675, 1278

Stolte, A., Grebel, E. K., Brandner, W., \& Figer, D. F. 2002, A\&A, 394, 459 


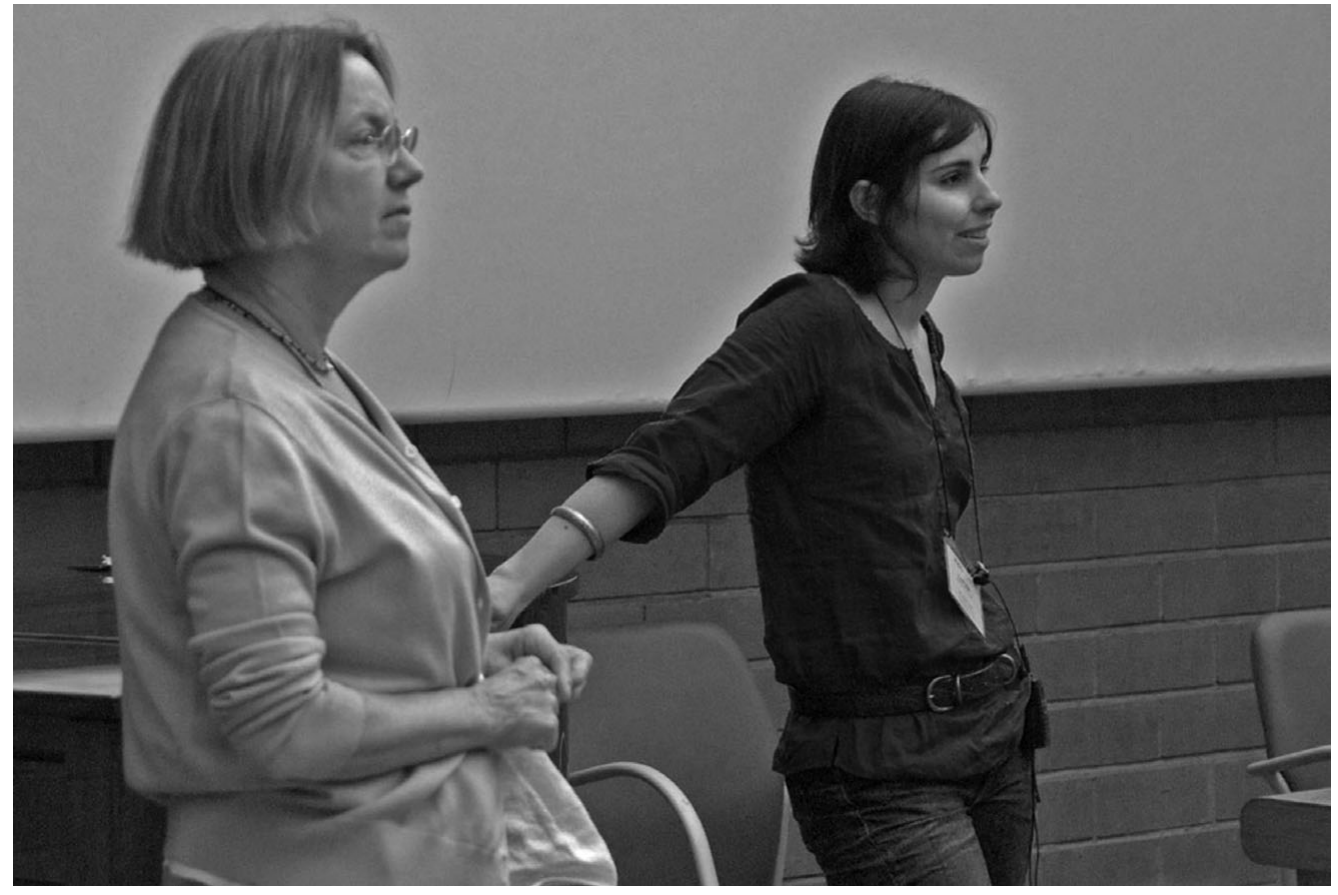

Lise Deharveng and Joana Ascenso 